\title{
Epidemiology of neoplastic colorectal polyps in a Caribbean country
}

\author{
SHAMIR O. CAWICH $^{1}$, AVIDESH MAHABIR ${ }^{1}$ and MILTON ARTHURS ${ }^{2}$ \\ ${ }^{1}$ Department of Clinical Surgical Sciences, University of The West Indies, St. Augustine Campus, St. Augustine, \\ Trinidad and Tobago; ${ }^{2}$ Department of Medicine, University of The West Indies, Mona Campus, Kingston, Jamaica
}

Received May 21, 2021; Accepted July 27, 2021

DOI: $10.3892 / \mathrm{mi} .2021 .10$

\begin{abstract}
Screening is practiced to identify and remove neoplastic colorectal polyps prior to their transformation into colorectal cancer (CRC). The aim of the present study was to document the epidemiology of neoplastic colorectal polyps in order to obtain important data that may then be used to guide screening protocols in Jamaica. For this purpose, an audit was performed to identify all consecutive patients who had neoplastic polyps detected at a screening colonoscopy at a facility in Jamaica from January 1, 2015 to December 30, 2018. The following data were collected: Patient demographics, polyp location, polyp synchronicity and histopathological information. The results revealed that a total of 480 colonoscopies were performed over the study period. With the exclusion of 2 patients with innumerable polyps as a part of polyposis syndrome, there were a total of 92 neoplastic polyps in 68 patients. Polyps were most commonly located in the right colon (55.6\%), followed by the left colon $(38 \%)$ and rectum $(6.5 \%)$. Upon the histological evaluation, 63 polyps were found to be benign adenomas with mild to moderate dysplastic alterations, 15 were adenomas with severe dysplasia and/or carcinoma in situ and 14 had foci of invasive carcinomas. On the whole, the present study demonstrates that $\sim 15 \%$ of the patients screened had neoplastic polyps that were recognized as precursor lesions for CRC. The majority of these were in the right colon. These results support the call for policy makers to institute national CRC screening programs, such as the National Comprehensive Cancer Network harmonized guidelines for the Caribbean.
\end{abstract}

\section{Introduction}

There is a well-documented association between neoplastic polyps and colorectal cancer (CRC) in the adenoma-carcinoma sequence $(1,2)$. Therefore, the majority of authorities

Correspondence to: Professor Shamir O. Cawich, Department of Clinical Surgical Sciences, University of The West Indies, St. Augustine Campus, St. Augustine, Trinidad and Tobago

E-mail: socawich@hotmail.com

Key words: cancer, colorectal, polyps, Caribbean, demographic recommend screening to identify and remove neoplastic polyps prior to their transformation into invasive CRC (3-7). However, the majority of Caribbean countries still do not have policies in place for universal CRC screening. This is largely due to the lack of prioritization by policy makers, resource limitations in the underfunded healthcare systems and a paucity of regional data to support CRC screening (8).

The present study thus aimed to document the epidemiology of neoplastic colorectal polyps and to obtain important data that may be used to guide screening protocols in Jamaica. To the best of our knowledge, there has been no prior report on the distribution or prevalence of neoplastic colorectal polyps in the Jamaican population.

\section{Patients and methods}

Patient information. After securing permission from the University of the West Indies Institutional Review Board (SA.1030/06/2021), a retrospective audit of hospital records was performed to identify all consecutive patients who underwent screening colonoscopy at the University Hospital of the West Indies in Kingston, Jamaica between January, 1, 2015 and December 30, 2018. Patient consent was waived by the local institutional review board. Only patients who underwent screening colonoscopies were included in the final study population. Patients who had colonoscopies for gastrointestinal-related symptomatology and those who had received procedures by trainee endoscopists were excluded from the study population. Patients who had polyps identified at colonoscopy were selected for further analysis. Patients with non-neoplastic polyps and those with incomplete clinical records were excluded from the study.

The hospital records of the patients were retrieved and the following data were extracted: Patient demographics, polyp location, polyp synchronicity and histopathological data.

\section{Results}

There were 480 colonoscopies performed over the study period using an Olympus 160 series colonoscope (Olympus Corporation). A standardized bowel preparation technique was used that included clear fluids for $12 \mathrm{~h}$, three sachets of PicoPrep ${ }^{\circledR}$ (sodium picosulphate) at 6-h intervals and two Dulcolax (Bisacodyl) tablets $6 \mathrm{~h}$ prior to the colonoscopy. 
These screening colonoscopies were all performed by expert gastroenterologists who were trained in accredited post-graduate fellowship programs. The caecum was intubated in $456(95 \%)$ cases and no complications were recorded in these cases.

These patients were deemed average-risk undergoing screening. There was a family history of colorectal carcinoma in a first degree relative in $11(2.3 \%)$ patients, a history of inflammatory bowel disease in $2(0.4 \%)$ patients and none with a past personal history of malignancies, lynch syndrome or familial adenomatous polyposis coli $(0 \%)$.

Of the total of 480 colonoscopies, 395 patients without polyps and 15 patients with non-neoplastic polyps were excluded from further analysis, leaving a total of 70 patients with neoplastic polyps in the study population. The clinicopathological features of the patients included in the study are presented in Table I. These patients were of Afro-Caribbean $(n=67)$, Caucasian $(n=2)$ and Chinese $(n=1)$ ethnicity. There were 37 males and 33 females with a mean age of 65.9 years (range, 29-88; median, 65; SD, \pm 11.65 ). A representation of the patient demographics by age is presented in Fig. 1.

In total, 45 patients had a solitary polyp and synchronous polyps were present in 25 patients: 22 patients had 2 polyps, 1 patient had 3 polyps and there were two females in the study with multiple polyps throughout the colon as a part of a polyposis syndrome. The number of polyps in the 2 patients with polyposis syndrome could not be counted accurately; thus, they were excluded from further analysis. This left a total number of 92 neoplastic polyps in 68 patients as the final study population.

The distribution of neoplastic polyps throughout the colon in these patients is illustrated in Fig. 2. The most common location was the right side $(55.6 \%)$, followed by left-sided lesions (38\%) and then the rectum (6.5\%). Upon the histological evaluation, 63 polyps were found to be benign adenomas with mild to moderate dysplastic alterations and 23 of these had a villous morphology. These polyps had a mean size of $1.02 \pm 0.85 \mathrm{~cm}$. There were 15 adenomas with high-grade dysplasia, 10 of which had a villous morphology. These 15 adenomas had a mean size of $1.8 \pm 1.28 \mathrm{~cm}$. In total, 14 polyps had foci of invasive carcinomas and all 14 had a villous morphology. These polyps were larger in size with a mean diameter of $2.57 \pm 0.61 \mathrm{~cm}$. The distribution of neoplastic polyps according to their histological evaluation is presented in Table II.

\section{Discussion}

The majority of authorities recommend CRC screening to detect neoplastic polyps when there is still an opportunity for polypectomy prior to their progression into invasive cancer (3-7). However, there was still only opportunistic screening in Jamaica up to the year 2021, despite an estimated incidence of CRC of 12 per 100,000 individuals in the population (8).

Previous studies have documented a 'right shift' in the Jamaican population (8-10) and in other Caribbean territories (11-13), where CRC primaries have become more prevalent in the right colon. Therefore, it was predictable that the precursor neoplastic polyps were most prevalent in the right colon in the present study. This is a change in disease
Table I. Clinicopathological characteristics of the patients in the present study $(n=70)$.

\begin{tabular}{lc}
\hline Characteristic & No. of patients \\
\hline Male & 37 \\
Female & 33 \\
Age, mean \pm SD (years) & $65.9 \pm 11.65$ \\
1 Polyp & 45 \\
2 Polyps & 22 \\
3 Polyps & 1 \\
Numerous polyps & 2 (excluded from \\
(polyposis syndrome) & the final analysis) \\
\hline
\end{tabular}

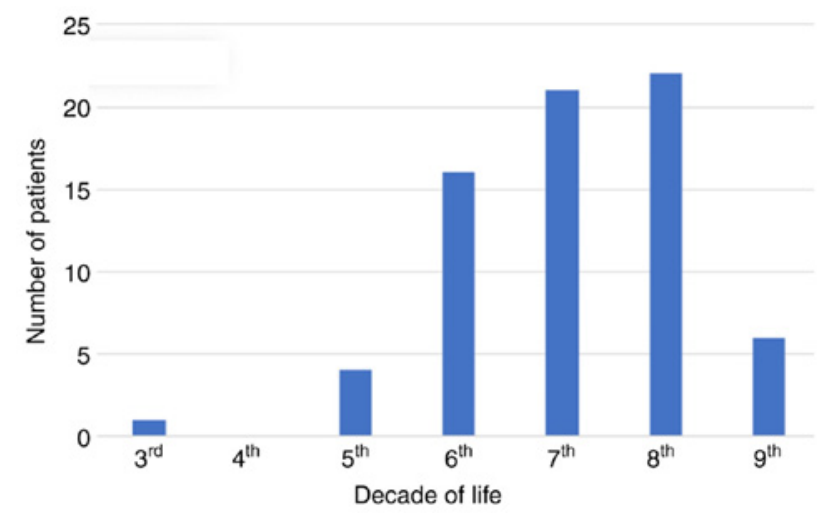

Figure 1. Distribution of patients with neoplastic polyps by patient age.

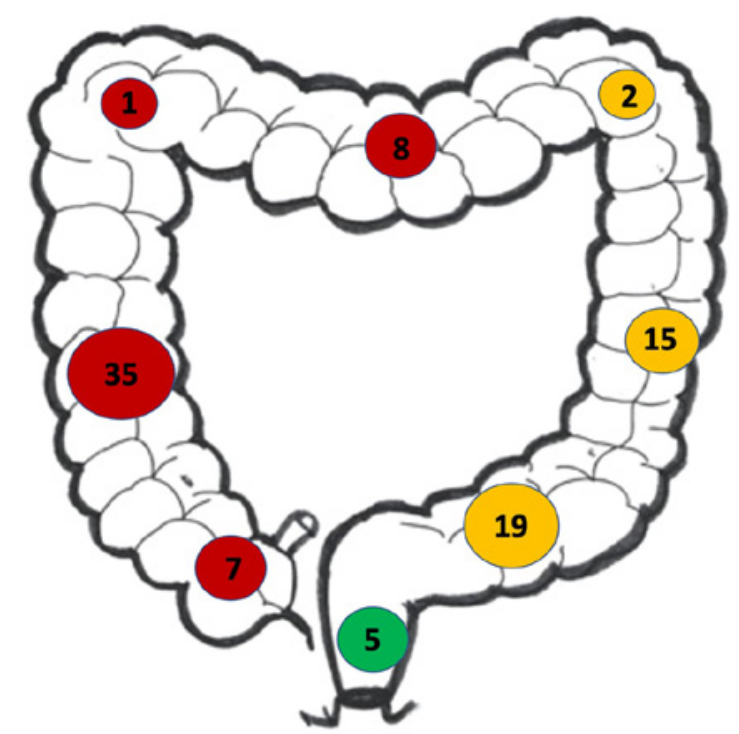

Figure 2. Distribution of neoplastic polyps throughout the colon and rectum. Red circles indicate the number of polyps in the right colon, yellow circles indicate the number of polyps in the left colon and the green circles indicates the number of polyps in the rectum.

distribution compared to studies from the 20th century, where CRC was predominantly left-sided $(14,15)$. In that era, some experts advocated sigmoidoscopy for screening. However, it is clear that a colonoscopy is required in modern screening 
Table II. Histological evaluation of neoplastic polyps.

\begin{tabular}{lcccc}
\hline $\begin{array}{l}\text { Location of } \\
\text { polyp }\end{array}$ & $\begin{array}{c}\text { All patients } \\
(\mathrm{n}=92), \mathrm{n}(\%)\end{array}$ & $\begin{array}{c}\text { No. of patients with adenomas with } \\
\text { low/intermediate grade dysplasia }\end{array}$ & $\begin{array}{c}\text { No. of patients with } \\
\text { carcinoma in situ }\end{array}$ & $\begin{array}{c}\text { No. of patients with } \\
\text { invasive carcinoma }\end{array}$ \\
\hline Right colon & $51(56)$ & 33 & 8 & 10 \\
Left colon & $36(39)$ & 26 & 7 & 3 \\
Rectum & $5(5)$ & 4 & 15 & 1 \\
Total & & 63 & 0 & 14 \\
\hline
\end{tabular}

programs, since neoplastic polyps and CRC are more common in the right colon.

The 'right side' shift cannot be overemphasized as there are fundamental differences in the clinical behavior of lesions, depending on the geographical origin in the colon (16). Right-sided CRCs have a high association with microsatellite instability and tend to present late, with non-specific symptoms (13), a higher cancer-related mortality rate (18-20) and worse overall survival statistics $(17,20)$.

It was not surprising that the present study found a male predominance for neoplastic polyps (1.1:1) as previous studies have reported a predominance for invasive CRC among males $(11,13,21)$. The demographic distribution was also predictable with a peak in the 6th to 8th decades of life. This matches the age distribution for invasive CRC in the Caribbean literature (8-13). However, it is notable that $7 \%$ of patients with neoplastic polyps were $<49$ years of age. In the possibility that the traditional recommendations for screening had commenced at the age of 50 years (4) had been followed, these polyps would have been missed (two of which had already undergone malignant alterations), as these polyps had evidently been formed at an earlier age. This was slightly lower than similar data reported from Barbados, where $10.3 \%$ of CRC diagnoses were made in individuals $<49$ years of age (13). These data lend support to the updated recommendations for CRC screening to begin at the age of 45 years in the Caribbean population (5). These are important data as there is an established association between a young age at diagnosis and aggressive disease, a higher CRC stage (22) and lower survival rates $(17,22)$. Although it is only a small proportion of the overall study group, it is still an important public health message that $7 \%$ of Jamaicans with precursor neoplastic polyps will be $<49$ years of age.

It should be noted that $96 \%$ of the patients in the present study were of Afro-Caribbean ethnicity. This was expected, since the matriline of the Jamaican population is almost entirely of West African descent (23). It is well-established that when individuals from the African diaspora develop CRC, they have a more aggressive tumor biology $(17,22,24,25)$, a greater association with KRAS mutations $(17,26)$ and an increased cancer-related mortality $(22,24)$. There is still an ongoing debate on whether these differences are due to a lack of screening, oncology care access inequities, socioeconomic factors or genuine differences in tumor biology; however, the fact that this is a predominantly Afro-Caribbean population must be recognized.

The present study documented the epidemiology of neoplastic colorectal polyps and demonstrated that these precursor lesions for CRC were identified in $15 \%$ of individuals who underwent colonoscopies. It is considered that these results will lend support to the call for policy makers to implement national screening protocols $(3,5)$. In the year 2018 , the Caribbean Association of Hematology Oncology facilitated a consensus meeting of Caribbean experts to create guidelines for CRC tailored to the Caribbean environment. These guidelines were endorsed by the National Comprehensive Cancer Network (NCCN) in the NCCN Harmonized Guidelines for the Caribbean (5). Thus, perhaps the time has come for Jamaican policy makers to implement these protocols.

In conclusion, the present study demonstrated that $\sim 15 \%$ of individuals who underwent colonoscopies had neoplastic polyps that were as recognized precursor lesions for CRC. There is also a predominance in right-sided polyps, with $56 \%$ of neoplastic colorectal polyps being found in the right colon. These results support the call for policy makers to institute national CRC screening programs, such as the NCCN harmonized guidelines for the Caribbean.

\section{Acknowledgements}

Not applicable.

\section{Funding}

No funding was received.

\section{Availability of data and materials}

The datasets used and/or analyzed during the current study are available from the corresponding author on reasonable request.

\section{Authors' contributions}

SOC, MA and AM conceptualized and designed the study. $\mathrm{AM}$ and MA collected the data and carried out the literature search. SOC, AM and MA analyzed the data. SOC prepared the manuscript. MA, AM and SOC edited the manuscript and approved the academic/intellectual content within the final version. All authors have read and approved the final manuscript. SOC, MA and AM confirm the authenticity of the data.

\section{Ethics approval and consent to participate}

In the present retrospective study, patient data were obtained after securing permission from the University of the West Indies Review Board (SA.1030/06/2021). 


\section{Patient consent for publication}

Not applicable.

\section{Competing interests}

The authors declare that they have no competing interests.

\section{References}

1. Mamlouk S, Simon T, Tomás L, Wedge TC, Arnold A, Menne A, Horst D, Capper D, Morkel M, Posada D, et al: Malignant transformation and genetic alterations are uncoupled in early colorectal cancer progression. BMC Biol 18: 116, 2020.

2. Lang NM, Kuipers J, Misselwitz B and Beerenwinkel N: Predicting colorectal cancer risk from adenoma detection via a two-type branching process model. PLoS Comput Biol 16: e1007552, 2020.

3. Pan American Health Organization (PAHO): Colorectal Cancer Screening in Latin America and the Caribbean. Strategies to reduce CRC incidence and mortality. PAHO Washington, DC, 2016. www.paho.org/hq/dmdocuments/2016/ Colorectal-Cancer-Screening-Landscape-English.pdf.

4. US Preventive Services Task Force; Bibbins-Domingo K, Grossman DC, Curry SJ, Davidson KW, Epling JW Jr, García FAR, Gillman MW, Harper DM, Kemper AR, Krist AH, et al: Screening for colorectal cancer: US preventive services task force recommendation statement. JAMA 315: 2564-2575, 2016.

5. National Comprehensive Cancer Network (NCCN): NCCN Harmonized Guidelines for the Caribbean: Colorectal Cancer. NCCN, Plymouth Meeting, PA, 2018. www.nccn. org/store/login/login.aspx?ReturnURL=https:/www.nccn. org/professionals/physician_gls/pdf/colon_harmonized-caribbean.pdf.

6. Meester RGS, Peterse EFP, Knudsen AB, de Weerdt AC, Chen JC, Lietz AP, Dwyer A, Ahnen DJ, Siegel RL, Smith RA, et al: Optimizing colorectal cancer screening by race and sex: Microsimulation analysis II to inform the American Cancer Society colorectal cancer screening guideline. Cancer 124: 2974-2985, 2018

7. Buskermolen M, Cenin DR, Helsingen LM, Guyatt G, Vandvik PO, Huag U, Bretthauer M and Lansdorp-Vogelaar I: Colorectal cancer screening with faecal immunochemical testing, sigmoidoscopy or colonoscopy: A microsimulation modelling study. BMJ 367: 15383, 2019.

8. Lee M, Brown EF, Mills MO and Walters CA: Colon cancer screening: Knowledge and attitudes in a Jamaican population and physicians. World J Research Rev 4: 4-7, 2018.

9. McFarlane MEC, Rhoden A, Fletcher PR and Carpenter R: Cancer of the colon and rectum in a Jamaican population: Diagnostic implications of the changing frequency and subsite distribution. West Ind Med J 53: 170-173, 2004.

10. Plummer JM, Leake PA, Ferron-Boothe D, Roberts PO, Mitchell DI and McFarlane ME: Colorectal cancer survival in Jamaica. Ann Med Surg (Lond) 6: 26-29, 2016.
11. Joachim C, Veronique-Baudin J, Razanakaivo M, Macni J, Pomier A, Dorival MJ, Smith-Ravin J, Pierre-Louis O and Escarmant P: Trends in colorectal cancer in the Caribbean: A population-based study in Martinique, 1982-2011. Rev Epidemiol Sante Publique 65: 181-188, 2017.

12. Zbar AP, Inniss M, Prussia PR and Shenoy R: The changing distribution of colorectal cancer in Barbados: 1985-2004. Dis Colon Rectum 50: 1215-1222, 2001.

13. Griffith S, Padmore G, Phillips E, Ramkissoon SSK, Moore S, Walkes K, Gohar AA and Cawich SO: Colorectal cancer demographics in barbados. Med Int 1: 1-5, 2021.

14. Lee M, Martin A and Terry SI: Colonoscopy in Jamaica-a 12-year experience. West Ind Med J 38: 213-216, 1989.

15. Lee MG and Hanchard B: Management of colonic polyps by colonoscopic polypectomy. West Ind Med J 40: 81-85, 1991.

16. Baran B, Mert Ozupek N, Yerli Tetik N, Acar E, Bekcioglu O and Baskin Y: Difference between left-sided and right-sided colorectal cancer: A focused review of literature. Gastroenterol Res 11: 264-273, 2018.

17. Augustus GJ and Ellis NA: Colorectal cancer disparity in African Americans: Risk factors and carcinogenic mechanisms. Am J Pathol 188: 291-303, 2018.

18. Hansen IO and Jess PM: Possible better long-term survival in left versus right-sided colon cancer-a systematic review. Dan Med J 59: A4444, 2012.

19. Kim SE, Paik HY, Yoon H, Lee JE, Kim N and Sung MK: Sex and gender-specific disparities in colorectal cancer risk. World J Gastroenterol 21: 5167-5175, 2015.

20. Elsaleh H, Joseph D, Grieu F, Zeps N, Spry N and Lacopetta B: Association of tumour site and sex with survival benefit from adjuvant chemotherapy in colorectal cancer. Lancet 355 : $1745-1750,2000$.

21. Brenner H, Kloor M and Pox CP: Colorectal cancer. Lancet 383: 1490-1502, 2014.

22. Gabriel E, Attwood K, Al-Sukhni E, Erwin D, Boland P and Nurkin S: Age-related rates of colorectal cancer and the factors associated with overall survival. J Gastrointest Oncol 9: 96-110, 2018.

23. Deason ML, Salas A, Newman SP, Macaulay VA, Morrison E and Pitsiladis YP: Interdisciplinary approach to the demography of Jamaica. BMC Evol Biol 12: 24, 2012.

24. Ollberding NJ, Nomura AMY, Wilkens LR, Henderson BE and Kolonel LN: Racial/ethnic differences in colorectal cancer risk: The multiethnic cohort study. Int J Cancer 129: 1899-1906, 2011.

25. Askari A, Nachiappan S, Currie A, Latchford A, Stebbing J, Bottle A, Athanasiou T and Faiz O: The relationship between ethnicity, social deprivation and late presentation of colorectal cancer. Cancer Epidemiol 47: 88-93, 2017.

26. Staudacher JJ, Yazici C, Bul V, Zeidan J, Khalid A, Xia Y, Krett $\mathrm{N}$ and Jung B: Increased frequency of KRAS mutations in African Americans compared with Caucasians in sporadic colorectal cancer. Clin Transl Gastroenterol 8: e124, 2017.

This work is licensed under a Creative Commons Attribution-NonCommercial-NoDerivatives 4.0 International (CC BY-NC-ND 4.0) License. 